\title{
A low-cost lightweight jacket for the protection of chronically indwelling jugular catheters in rhesus monkeys
}

\author{
JANE J. TURNER and JAMES G. HERNDON \\ Division of Neurobiology, Yerkes Regional Primate Research Center \\ Emory University, Atlanta, Georgia 30322
}

\begin{abstract}
A jacket for long-term protection of chronically indwelling jugular catheters in rhesus monkeys is described. The jacket is made of lightweight, durable, and inexpensive material and is easily modified to fit animals of different sizes. It has been used successfully on over 25 animals.
\end{abstract}

Research currently in progress in our laboratory requires the use of rhesus monkeys with chronically implanted jugular catheters. Catheters are routed subcutaneously to an exit point in the skin of the cervical region of the animal's back. The external portion of the catheter and the skin opening must be continuously protected by a jacket.

Although lightweight jackets of porous material are commercially available, their high cost limits the number of experimental animals that can be used simultaneously. Jackets designed primarily to carry animal-worn instrumentation (Karl \& Kissen, 1978) are generally too bulky to permit optimal animal comfort during prolonged periods of wear. We therefore designed a catheterprotection jacket that is inexpensive, easy to produce, and constructed of light, porous, and durable material. The jacket can readily be tailored to fit animals of varying sizes. Details for construction are provided below.

\section{MATERIALS}

Fabric used for the jacket is machine washable, $65 \%$ cotton $/ 35 \%$ polyester canvas, similar to lightweight denim. The raw edges are bound with polyester/cotton double-fold bias tape; the back closure is accomplished with a $25-\mathrm{cm}(10-\mathrm{in}$.) medium-weight metal separating jacket zipper. A small safety pin fastened across the closed zipper just above the pull prevents the animal's opening the zipper. Total cost of materials for one jacket is about $\$ 6$.

\section{CONSTRUCTION}

\section{Pattem Adjustments}

The pattern shown in Figure 1 can be adjusted to

This work was supported by NSF Grant 76-84314 and NIH Grant RR-00165. The authors wish to thank artist Brooke Holliman for preparing the pattern diagram. fit female and male rhesus monkeys weighing approximately $6-12 \mathrm{~kg}$. The solid lines represent a jacket for an animal of $8-9 \mathrm{~kg}$. To reduce the size of the jacket, more overlap of side seams and larger darts are required. The dotted lines represent the cutting line for large animals, providing larger arm and neck openings and greater chest and abdominal girth.

\section{Construction Procedure}

Care should be taken during the construction of the jacket to assure that no rough edges are left on the inside that might cause rubbing and abrasions. After the fabric has been cut, raw edges of the arm, neck, and zipper openings are bound with bias tape by placing the raw edge in the fold of the tape and zig-zag stitching the tape edge on each side of the fabric. The zipper is then sewn in place (with no folding of the material), with the closure at either the top or the bottom. Side seams are made by lapping one piece of fabric over the other and double stitching each edge, first with narrow and then with wider zig-zag stitches (Figure 1, insert). This provides a smooth, flat seam and prevents raveling of the fabric. The bottom edge of the completed jacket is bound with one long strip of bias tape.

The jacket can then be fitted to the tranquilized animal for determination of dart sizes. Waist size should be reduced enough to prevent the animal's pulling up or reaching under the jacket, while at the same time allowing room for comfort and normal expansion of the abdomen during eating and drinking. An extra 1 in. or so larger than the animal's waist is appropriate. Dart size is easily changed later to accommodate weight gain or loss.

The arm and neck openings are the points at which the likelihood of discomfort and abrasions is greatest, so particular care should be taken to assure that they are large enough. If the skin in these areas is pink after the animal has worn the jacket for 1 day or so, the holes can be cut larger and refinished with binding tape. It is not necessary to take out the side seams for this modification. 


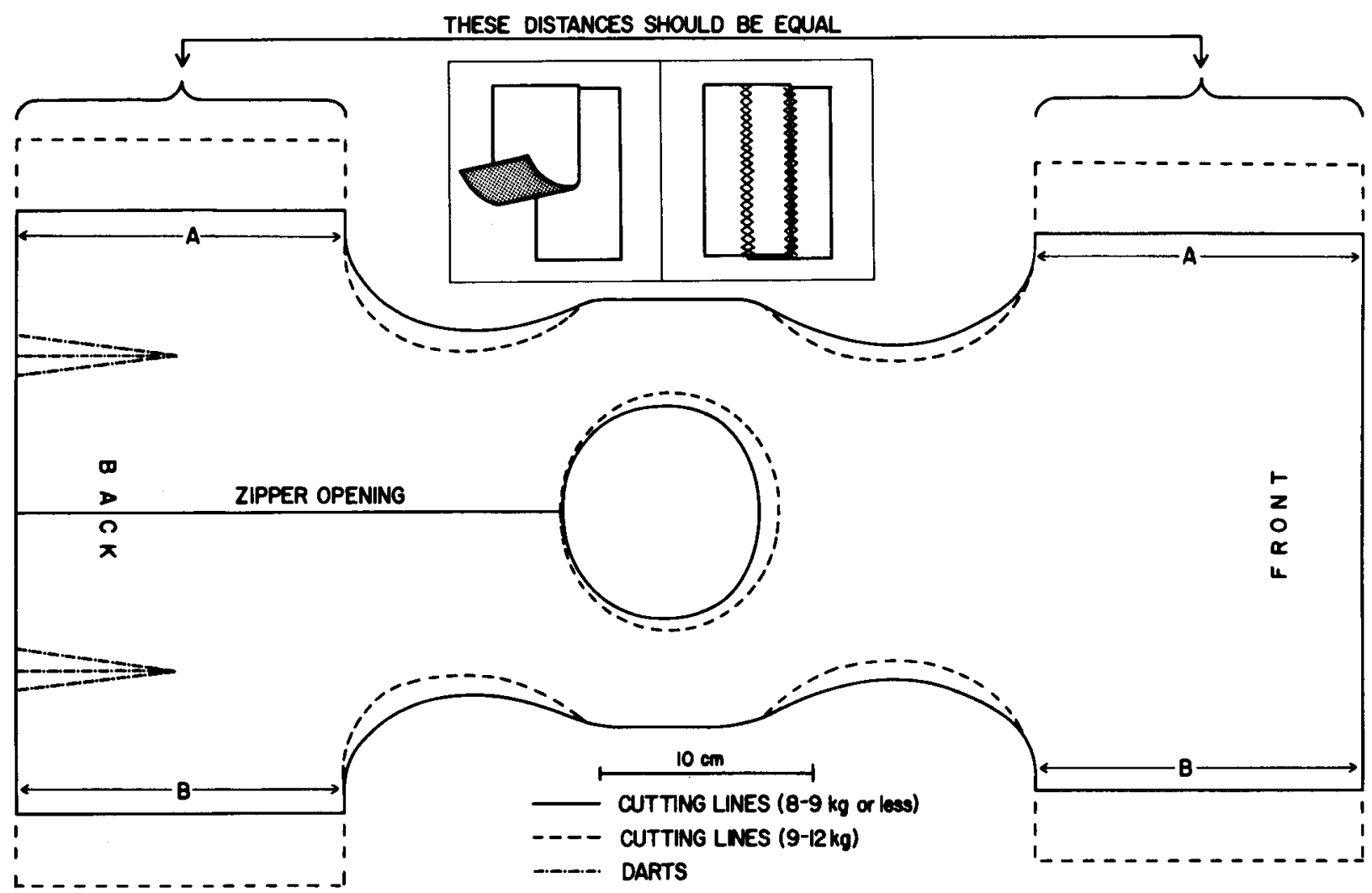

Figure 1. Pattern for catheter-protection jacket. Side seams are constructed by sewing Lines A and B on front panel to likelettered lines on back panel. Method of seam closure is illustrated in insert (wrong side of fabric is shaded).

Time required for the construction of a single jacket is about $2 \mathrm{~h}$. Figure 2 shows the front and back of the finished jacket.

\section{Repairs}

Repairs of worn areas or small holes and rips can be accomplished easily by cutting a piece of material large enough to cover the damaged area and zig-zag stitching it in place on the surface of the jacket.

The jacket has provided adequate protection for chronic venous catheters in more than 25 animals. The low cost is a significant advantage over commercially available models, and durability is not compromised. An

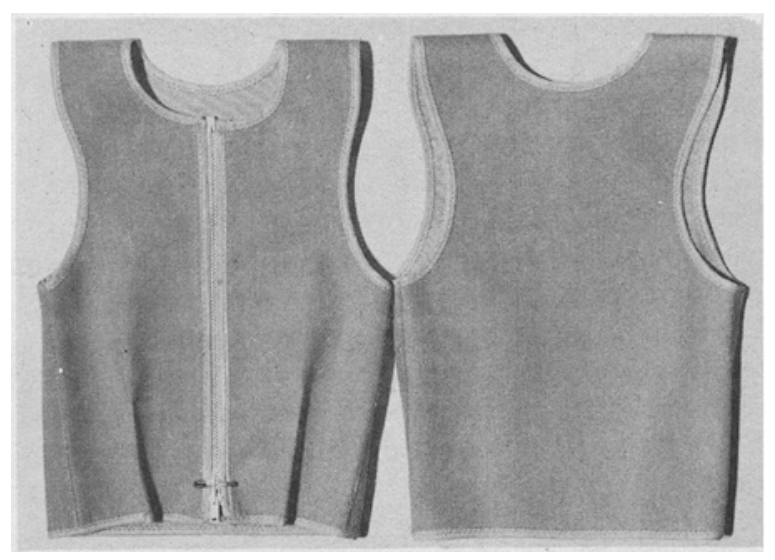

Figure 2. Front and back view of completed jacket. active adult male, living outdoors with other animals, can wear the same jacket with only minor repairs for more than 1 year.

The lightweight and porous texture of the fabric appears to maximize animal comfort. Most monkeys adapt readily to the jacket and seem completely unencumbered by its presence. However, a small proportion of animals (about 10\%) have been intolerant of the jacket to the point that it did not seem feasible to persist in attempting to adapt them to its wear. The behavior of these animals included constant biting and pulling at the jacket and generally increased activity levels that we felt were indicative of stress. Such animals

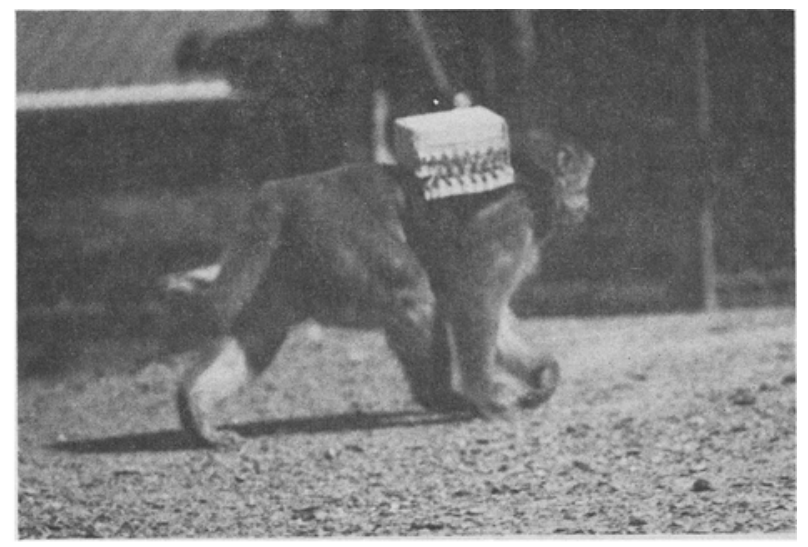

Figure 3. Jacket with backpack for instrumentation in place. 
were subsequently dropped from experiments that would necessitate jacket wear. We have therefore found it expedient to institute jacket wear several days prior to catheter implantation in order to assess the animal's response. Males used in our research have had their canine teeth blunted, which may minimize jacket damage if biting occurs.

Our use of catheterized animals often requires that animals carry an instrumentation backpack weighing $1,100 \mathrm{~g}$ (Herndon, Perachio, Collins, \& Turner, 1979). The catheter-protection jacket described here can be modified to enable the animal to carry this backpack comfortably. A pouch designed to accommodate the instrumentation unit is sewn to the jacket on one side of the zipper and fastened with a shoelace and eyelets to the other side (Figure 3).

\section{REFERENCES}

Herndon, J. G., Perachio, A. A., Collins, D. C., \& Turner, J. J. Testosterone levels during sexual behavior of rhesus monkeys as measured in samples collected by an animal-worn blood sampling device. The Physiologist, 1979, 22, 55. (Abstract)

Karl, A. A., \& Kissen, A. T. Durable jacket for nonhuman primates to protect chronically implanted instrumentation. Laboratory Animal Science, 1978, 28, 103-105.

(Received for publication October 2, 1980; accepted October 13, 1980.) 